Report no. 06/16

\title{
The Kink Phenomenon in Fejér and Clenshaw-Curtis Quadrature
}

\author{
J. A. C. Weideman \\ University of Stellenbosch \\ Applied Mathematics \\ L. N. Trefethen \\ Oxford University Computing Laboratory
}

The Fejér and Clenshaw-Curtis rules for numerical integration exhibit a curious phenomenon when applied to certain analytic functions. When $N$ (the number of points in the integration rule) increases, the error does not decay to zero evenly but does so in two distinct stages. For $N$ less than a critical value, the error behaves like $O\left(\varrho^{-2 N}\right)$, where $\varrho$ is a constant greater than 1 . For these values of $N$ the accuracy of both the Fejér and ClenshawCurtis rules is almost indistinguishable from that of the more celebrated Gauss-Legendre quadrature rule. For larger $N$, however, the error decreases at the rate $O\left(\varrho^{-N}\right)$, i.e., only half as fast as before. Convergence curves typically display a kink where the convergence rate cuts in half. In this paper we derive explicit as well as asymptotic error formulas that provide a complete description of this phenomenon.

Key words and phrases: Fejér, Clenshaw-Curtis, Gauss quadrature; convergence rate

This work was supported by the Royal Society of the UK and the National Research Foundation of South Africa under the South Africa-UK Science Network Scheme. The first author also acknowledges grant FA2005032300018 of the NRF.

Oxford University Computing Laboratory

Numerical Analysis Group

Wolfson Building

Parks Road

Oxford, England OX13QD

E-mail: LNT@comlab.oxford.ac.uk

September, 2006 


\section{Introduction}

In a recent paper some remarkable convergence properties of the Clenshaw-Curtis rule for numerical integration were pointed out [15]. To elucidate this behaviour we consider one of the examples from that paper, namely

$$
I=\int_{-1}^{1} \frac{d x}{1+16 x^{2}}
$$

Figure 1 shows the absolute errors when this integral is approximated by the $N$ point Fejér, Clenshaw-Curtis, and Gauss quadrature rules. (Here, and throughout, the expression "Gauss quadrature" refers to the Gauss formula associated with constant weight function, i.e., the well-known Gauss-Legendre quadrature. Perhaps less familiar to nonspecialists are the Fejér and Clenshaw-Curtis rules and hence we define these two rules below.) Note that Figure 1 shows logarithmic scales on the vertical axes: straight lines therefore represent geometric convergence rates.

The Gauss rule, in particular, converges according to $O\left(\varrho^{-2 N}\right)$, where

$$
\varrho=\frac{1}{4}(1+\sqrt{17})=1.28 \ldots
$$

which is consistent with theory. The interesting behavior is exhibited by the Fejér and Clenshaw-Curtis curves. For large $N$ both these rules converge according to $O\left(\varrho^{-N}\right)$, i.e., half as fast as the Gauss rule, which is also consistent with existing theory. The remarkable observation of [15] is the fact that for smaller values of $N$ the Fejér and Clenshaw-Curtis rules converge not as $O\left(\varrho^{-N}\right)$ but as $O\left(\varrho^{-2 N}\right)$. That is, the initial convergence rate is twice the ultimate convergence rate and the error curves display a kink where the convergence switches from one rate to the other. This means that initially both these rules converge about as fast as the Gauss rule. Indeed, in Figure 1 there is negligible distinction between the three error curves for smaller values of $N$.

The purpose of this paper is to present a quantitative explanation of this phenomenon. Among other things, we shall derive equations for computing the critical values of $N$ shown in Figure 1 . We do this by deriving explicit formulas for the error curves. These error formulas consist of two main terms, one of which dominates for values of $N$ less than the critical one and the other of which dominates for larger $N$ (see Theorem 7). Our characterization of the kink will come from setting these two terms equal.

The results shown in Figure 1 correspond to even values of $N$. For odd $N$ the kinks occur at smaller values of $N$, as can be seen in [15]. This fact will also be explained by our analysis.

For the sake of completeness we start with a quick summary of the basic formulas of interpolatory quadrature for integrals of the form

$$
I(f)=\int_{-1}^{1} f(x) d x
$$


(a) Fejer

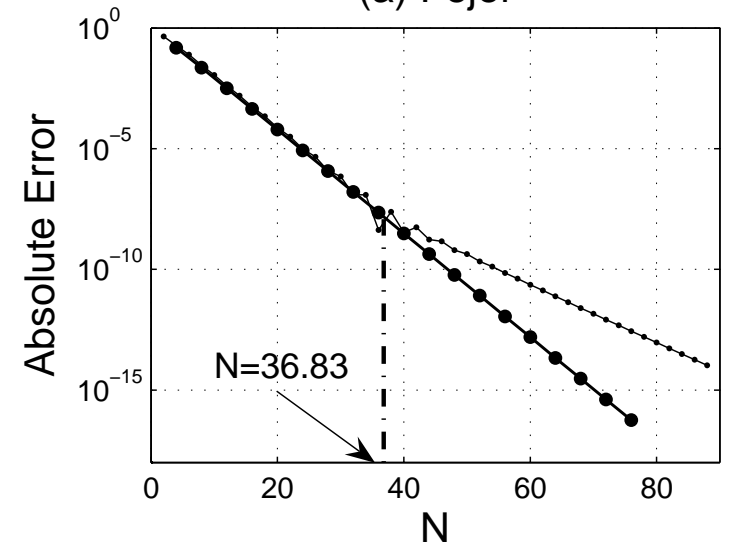

(b) Clenshaw-Curtis

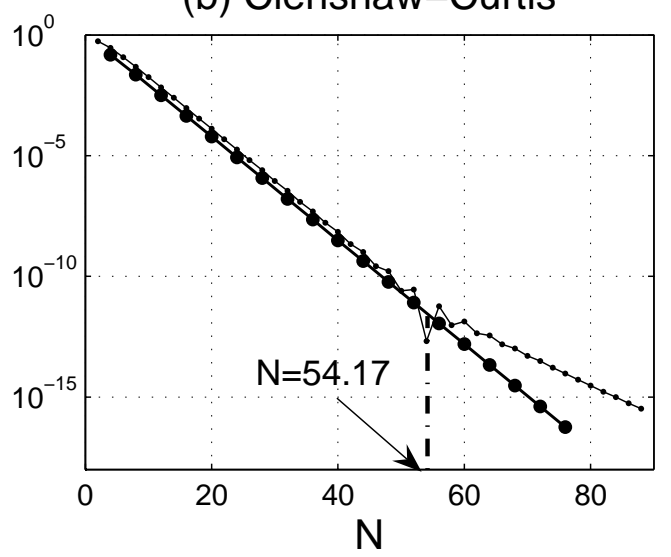

Figure 1: Absolute errors when approximating the integral (1.1) with various $N$-point interpolatory quadrature rules ( $N$ even). The tiny dots represent (a) Fejér and (b) Clenshaw-Curtis quadrature while the large dots represent Gauss quadrature. The Fejér and Clenshaw-Curtis convergence rates start out the same as for Gauss, but at a certain value of $N$ (indicated by the vertical dash-dot line) a kink appears and the rate cuts in half. The critical values of $N$ are the solutions to the equations (3.10) and (3.11).

Let $\left\{x_{j}\right\}_{j=1}^{N}$ be a set of distinct nodes in $[-1,1]$ and define

$$
\omega_{N}(x)=c \prod_{j=1}^{N}\left(x-x_{j}\right)
$$

where $c$ is a non-zero normalization constant. (Its value is immaterial as it will cancel in all the formulas below.) Let $p_{N-1}(x)$ be the polynomial of degree $N-1$ that interpolates the function $f(x)$ at the nodes $\left\{x_{j}\right\}_{j=1}^{N}$. This interpolating polynomial can be expressed in Lagrange form as

$$
p_{N-1}(x)=\sum_{j=1}^{N} \frac{\omega_{N}(x)}{\omega_{N}^{\prime}\left(x_{j}\right)\left(x-x_{j}\right)} f\left(x_{j}\right) .
$$

An interpolatory quadrature rule is derived by integrating this expression. That is, we approximate $I(f)$ by $I_{N}(f)$, where

$$
I_{N}(f) \equiv \int_{-1}^{1} p_{N-1}(x) d x=\sum_{j=1}^{N} w_{j} f\left(x_{j}\right),
$$

with weights defined by

$$
w_{j}=\frac{1}{\omega_{N}^{\prime}\left(x_{j}\right)} \int_{-1}^{1} \frac{\omega_{N}(x)}{x-x_{j}} d x .
$$

Two popular choices for the nodes are (a) the set of zeros of the Chebyshev polynomial of degree $N$, and (b) the set of extrema (including endpoint extrema) of the 
Chebyshev polynomial of degree $N-1$. The corresponding quadrature rules are named after (a) Fejér, and (b) Clenshaw and Curtis. We summarize as follows (with $T_{N}(x)$ denoting, as usual, the Chebyshev polynomial of degree $N$ ):

$$
\text { Fejér: } \quad \omega_{N}(x)=T_{N}(x), \quad x_{j}=\cos \left(\frac{(2 j-1) \pi}{2 N}\right),
$$

and

$$
\text { Clenshaw-Curtis: } \quad \omega_{N}(x)=\left(1-x^{2}\right) T_{N-1}^{\prime}(x), \quad x_{j}=\cos \left(\frac{(j-1) \pi}{N-1}\right) .
$$

In both cases the weights $w_{j}$ can be computed explicitly; the formulas are given in [3, Sect. IV.5] and [6, Sect. 2.5.5]. (We remark that the Fejér rule defined above is more precisely called Fejér's first rule; for Fejér's second rule see [6, Sect. 2.5.5]. The first and second Fejér rules are sometimes called the Pólya and Filippi rules, respectively; see [3, Ch. IV].)

The most famous choice of nodes is the one leading to Gauss quadrature. Here $\omega_{N}(x)=P_{N}(x)$, the Legendre polynomial of degree $N$. Compared with the rules based on Chebyshev points this rule has the disadvantage that the nodes are not given by any explicit formula and some form of iteration is required for their computation. On the other hand, it has a distinct advantage in the fact that the $N$-point Gauss rule exactly integrates polynomials of degree up to $2 N-1$ (inclusive). By contrast, the $N$-point Fejér and Clenshaw-Curtis rules both have the property of exactness for polynomials of degree up to $N-1$ only. The $2 N-1$ vs. $N-1$ superiority of the Gauss rule gives it the factor of two advantage in speed of convergence that we alluded to above. (Note that this advantage applies only to analytic functions; for nonanalytic functions there may be no advantage [15].)

The outline of the paper is as follows. In Section 2 we derive an explicit error formula for interpolatory quadrature rules in the case where the integrand is a rational function. In particular, we obtain explicit formulas for the error curves shown in Figure 1 . The formulas derived in this section do not immediately lend themselves to a characterization of the kink phenomenon, and further manipulation and asymptotic estimation are required. This is done in Section 3, where we derive the equations for computing the critical values of $N$. The key step here is a new formula for an integral involving the Chebyshev polynomials that arises quite often in numerical analysis. In Section 4 we introduce the idea of the kink curve, which can be described as follows. Consider a model function and its integral

$$
f_{z}(x)=\frac{1}{z-x}, \quad I\left(f_{z}\right)=\int_{-1}^{1} f_{z}(x) d x,
$$

where $z$ is a complex number not in $[-1,1]$. When Fejér or Clenshaw-Curtis quadrature is used to approximate this integral, the critical $N$ where the kink occurs depends on the position of the singularity $z$. We define the kink curve as the locus of all $z$ for which the kink appears at the same value of $N$. A perturbation analysis will be used to show that this curve is, in the limit as $N \rightarrow \infty$ through the even integers, an ellipse with semiaxes 1 and $2 \log N / N$ (Fejér) and semiaxes 1 and $3 \log N / N$ (Clenshaw-Curtis). 


\section{Error Formula for the Model Problem}

Here we derive an explicit formula for the error in any approximation to the model integral (1.7) based on interpolatory quadrature. This result is not new (similar error formulas can be found, for example, in [3, Ch. 4]), but we nevertheless state our own version of the formula as a theorem. The key to the proof is the Hermite contour integral representation of the error in polynomial interpolation; see [5, p. 68] or [10, p. 245].

Theorem 1 Let $f_{z}(x)$ and $I\left(f_{z}\right)$ be defined by (1.7). Let $I_{N}\left(f_{z}\right)$ denote the approximation to $I\left(f_{z}\right)$ as computed by the interpolatory quadrature rule based on the nodal set $\left\{x_{j}\right\}_{j=1}^{N}$. Then

$$
I\left(f_{z}\right)-I_{N}\left(f_{z}\right)=\frac{E_{N}(z)}{\omega_{N}(z)}
$$

where

$$
E_{N}(z)=\int_{-1}^{1} \frac{\omega_{N}(x)}{z-x} d x
$$

Proof Let $p_{N-1}(x)$ be the polynomial interpolant of $f_{z}(x)$ at the nodes $\left\{x_{j}\right\}_{j=1}^{N}$. According to the Hermite formula, the pointwise error is given by

$$
f_{z}(x)-p_{N-1}(x)=\frac{\omega_{N}(x)}{2 \pi i} \int_{C} \frac{d \zeta}{\omega_{N}(\zeta)(\zeta-x)(z-\zeta)},
$$

where $\omega_{N}(x)$ is defined by (1.3), and $C$ is a simple closed rectifiable curve that encloses the interval $[-1,1]$ but excludes the pole at $\zeta=z$. (Proof: Evaluate the integral by residues and use (1.4).)

Observe that the integrand on the right side of (2.3) is a rational function with the denominator degree exceeding the numerator degree by at least 2 . If one therefore deforms the contour $C$ into a circle $|\zeta|=R$, the integral will vanish in the limit $R \rightarrow \infty$ [1, Sect. 4.2]. To adjust for the fact that the singularity at $\zeta=z$ is traversed in the process of deforming the contour its residue is subtracted and therefore

$$
f_{z}(x)-p_{N-1}(x)=\frac{\omega_{N}(x)}{\omega_{N}(z)} \frac{1}{z-x} .
$$

The quadrature error (2.1) follows by integrating this expression.

For any set of nodes $\left\{x_{j}\right\}_{j=1}^{N}$ it is possible, at least in principle, to compute the integral $E_{N}(z)$ defined in (2.2) since its integrand is a rational function. In the case of Gauss quadrature the formula is particularly simple. With $\omega_{N}(x)=P_{N}(x)$ one finds in many tables of integrals Neumann's formula,

$$
\int_{-1}^{1} \frac{P_{N}(x)}{z-x} d x=2 Q_{N}(z),
$$

where $Q_{N}$ is the Legendre function of the second kind. In the case of quadrature based on Chebyshev points, $\omega_{N}(x)$ is defined in (1.5)-(1.6). In the Clenshaw-Curtis case, the formula for $\omega_{N}(x)$ can be simplified by the identity [11, p. 35]

$$
\left(1-x^{2}\right) T_{N-1}^{\prime}(x)=\frac{N-1}{2}\left(T_{N-2}(x)-T_{N}(x)\right), \quad N \geq 2,
$$


and hence we define

$$
\text { Clenshaw-Curtis: } \quad \omega_{N}(x)=T_{N}(x)-T_{N-2}(x), \quad N \geq 2 .
$$

Both the Fejér and Clenshaw-Curtis cases will therefore require the evaluation of the integral

$$
S_{N}(z) \equiv \int_{-1}^{1} \frac{T_{N}(x)}{z-x} d x
$$

This integral, which is not as well documented as the corresponding one (2.4) for Legendre polynomials, holds the key to the kink phenomenon. In the next section we record a few known results regarding this integral and contribute a few formulas we believe to be new.

Using (2.4)-(2.5) we can now specialize Theorem 1 to the three quadrature rules considered in this paper.

Theorem 2 Let $f_{z}(x)$ and $I\left(f_{z}\right)$ be defined by (1.7). Let $G_{N}\left(f_{z}\right), F_{N}\left(f_{z}\right)$, and $C_{N}\left(f_{z}\right)$ denote, respectively, the $N$-point Gauss, Fejér, and Clenshaw-Curtis approximations to $I\left(f_{z}\right)$. Then

$$
I\left(f_{z}\right)-G_{N}\left(f_{z}\right)=2 \frac{Q_{N}(z)}{P_{N}(z)}
$$

and

$$
I\left(f_{z}\right)-F_{N}\left(f_{z}\right)=\frac{S_{N}(z)}{T_{N}(z)}, \quad I\left(f_{z}\right)-C_{N}\left(f_{z}\right)=\frac{S_{N}(z)-S_{N-2}(z)}{T_{N}(z)-T_{N-2}(z)},
$$

where $S_{N}(z)$ is defined by $(2.5)$.

We postpone a discussion of the large $N$ behavior of these error formulas to the next section; see Theorem 7 .

There is another reason for studying integrals such as (2.2) and (2.5). By explicitly computing the quantities on the left side of (2.1) we obtain

$$
\phi(z)-r_{N}(z)=\frac{E_{N}(z)}{\omega_{N}(z)},
$$

where

$$
\phi(z) \equiv \ln \left(\frac{z+1}{z-1}\right), \quad r_{N}(z) \equiv \sum_{j=1}^{N} \frac{w_{j}}{z-x_{j}} .
$$

The left side of (2.8) represents the error in approximating the function $\phi(z)$ by a rational function $r_{N}(z)$ of numerator degree $N-1$, denominator degree $N$, and poles at $\left\{x_{j}\right\}_{j=1}^{N}$. This idea is not new. Indeed, it goes back in essence to Gauss, whose invention of Gauss quadrature was based on the construction via continued fractions of rational functions that match $\phi(z)$ to as high an order as possible at $z=\infty$ [9]. Thus in the case of Gauss quadrature, $r_{N}(z)$ is the type $(N-1, N)$ Padé approximation to $\phi(z)$ at $z=\infty$. More explicit use of the approximation problem $r_{N}(z) \approx \phi(z)$ was made by Takahasi and Mori 
[14], who took this as the principle for analyzing and comparing all kinds of quadrature formulas and made contour plots of $\left|\phi(z)-r_{N}(z)\right|$ in the complex plane. It was contour plots like this for Clenshaw-Curtis quadrature, presented in [15], that led to the present paper.

We conclude this section by pointing out that Theorem 1 can be generalized to any rational function of the form

$$
r(x)=\sum_{k=0}^{K} a_{k} x^{k}+\sum_{k=1}^{M} \frac{b_{k}}{z_{k}-x}
$$

with all poles $z_{k}$ distinct. Indeed, for any $N$-point interpolatory rule with $N>K$ the quadrature error is given by

$$
I(r)-I_{N}(r)=\sum_{k=1}^{M} b_{k} \frac{E_{N}\left(z_{k}\right)}{\omega_{N}\left(z_{k}\right)}
$$

where $E_{N}(z)$ is defined by (2.2). (For Gauss quadrature only $N>K / 2$ is required.) By applying this result to the test function of Figure 1 explicit formulas for the error curves shown in that figure can be derived. This is summarized in the following example.

\section{Example 1 Consider}

$$
g_{a}(x) \equiv \frac{a^{2}}{a^{2}+x^{2}}=\frac{a i}{2}\left(\frac{1}{a i+x}+\frac{1}{a i-x}\right)
$$

An application of (2.9) yields

$$
I\left(g_{a}\right)-I_{N}\left(g_{a}\right)=a i \frac{E_{N}(a i)}{\omega_{N}(a i)}
$$

where we have assumed a symmetric node distribution, i.e., $\omega_{N}(x)$ is an even (resp. odd) function when $N$ is even (resp. odd). Therefore,

$$
I\left(g_{a}\right)-G_{N}\left(g_{a}\right)=2 a i \frac{Q_{N}(a i)}{P_{N}(a i)}
$$

and

$$
I\left(g_{a}\right)-F_{N}\left(g_{a}\right)=a i \frac{S_{N}(a i)}{T_{N}(a i)}, \quad I\left(g_{a}\right)-C_{N}\left(g_{a}\right)=a i \frac{S_{N}(a i)-S_{N-2}(a i)}{T_{N}(a i)-T_{N-2}(a i)}
$$

which define the error curves of Figure 1 (where $a=1 / 4$ ). 


\section{Formulas for $S_{N}(z)$}

In order to explain the kinks in Figure 1, our first priority is to examine the behaviour of the integral $S_{N}(z)$ that appears in the error formulas (2.7) and (2.10). We start by summarizing existing results from the literature. The main contribution of this section is a new expression for $S_{N}(z)$ that makes it possible to determine the location of the kink precisely.

In what follows $E_{\varrho}$ denotes the ellipse

$$
z=\frac{1}{2}\left(\xi+\xi^{-1}\right), \quad \xi=\varrho e^{i \theta}, \quad 0 \leq \theta \leq 2 \pi,
$$

with foci at $z= \pm 1$ and the sum of its semi-major and -minor axes equal to $\varrho(>1)$. This means that if $z \in E_{\varrho}$,

$$
\xi=z+\sqrt{z^{2}-1}
$$

with the branch cut of the square root taken to be consistent with $|\xi|>1$.

Lemma 3 (Chawla, 1968 [4]) Let $S_{N}(z)$ be defined by (2.5) with $z \in E_{\varrho}$; then

$$
S_{N}(z)=4 \xi^{-N-1} \sum_{k=-\lfloor N / 2\rfloor}^{\infty} \frac{(2 k+N+1) \xi^{-2 k}}{(2 k+2 N+1)(2 k+1)}
$$

where $\lfloor k\rfloor$ denotes the greatest integer less than or equal to $k$.

The following formula is more recent.

Lemma 4 (Notaris, $2006[12]$ ) For even $N$,

$$
S_{N}(z)=T_{N}(z) \ln \left(\frac{z+1}{z-1}\right)-4 \sum_{k=1}^{\lfloor(N+1) / 2\rfloor} \frac{T_{N-2 k+1}(z)}{2 k-1},
$$

while for odd $N$ the last term in the sum should be halved.

We derived the following formula independently of the above two lemmas. Since it can be obtained from Lemma 3 using partial fraction decomposition we omit the details of our derivation.

Lemma 5 Let $z \in E_{\varrho}$; then for even $N$

$$
S_{N}(z)=\frac{2}{\xi}\left(\sum_{k=0}^{\infty} \frac{\xi^{-2 k}}{2 k+N+1}+\sum_{k=0}^{\infty} \frac{\xi^{-2 k}}{2 k-N+1}\right) .
$$

For odd N,

$$
S_{N}(z)=\frac{1}{2 z}\left(S_{N+1}(z)+S_{N-1}(z)\right)
$$


(The formula for odd $N$ follows from inserting the three-term recurrence relation for Chebyshev polynomials into (2.5) and then proceeding as in [16].)

None of the above three lemmas is particularly suited for explaining the kink phenomenon. In fact, in [4] Chawla obtains a bound on the error in Clenshaw-Curtis quadrature by estimating the series in Lemma 3, which led to the remark "... these estimates are poor if $\varrho$ is near 1." Notaris makes a similar comment in the last paragraph of [12]. The error estimate for Clenshaw-Curtis quadrature presented by Brass [3, Satz 80] does not display a sharp kink either. Here is a new formula for $S_{N}(z)$ that gives a better estimate.

Lemma 6 Let $z \in E_{\varrho}$; then

$$
S_{N}(z)=\sigma \xi^{-N} \pi i+\left\{\begin{array}{cc}
W_{N}(\xi), & \text { Neven } \\
\frac{1}{2 z}\left(W_{N+1}(\xi)+W_{N-1}(\xi)\right), & \text { Nodd }
\end{array}\right.
$$

where $\sigma$ is defined by

$$
\sigma \equiv\left\{\begin{array}{rr}
-1, & \operatorname{Im} z>0 \\
0, & \operatorname{Im} z=0 \\
+1, & \operatorname{Im} z<0
\end{array}\right.
$$

and

$$
W_{N}(\xi) \equiv 2\left(\xi^{-N} \int_{0}^{\xi} \frac{w^{N}}{1-w^{2}} d w+\xi^{N} \int_{0}^{1 / \xi} \frac{w^{N}}{1-w^{2}} d w\right) .
$$

When $z$ is real (i.e., $\xi>1$ or $\xi<-1$ ) the first integral in (3.5) should be interpreted in the principal value sense.

Proof Consider the formula in Lemma 5, with $N$ even. Starting with the second series in (3.1), note that it can be split into a finite sum with negative integers in the denominator and an infinite series with positive integers. This yields

$$
\sum_{k=0}^{\infty} \frac{\xi^{-2 k}}{2 k-N+1}=-\xi^{1-N} \sum_{k=1}^{N / 2} \frac{\xi^{2 k-1}}{2 k-1}+\xi^{1-N} \sum_{k=0}^{\infty} \frac{\xi^{-(2 k+1)}}{2 k+1} .
$$

By manipulating geometric series both summations on the right can be expressed in terms of integrals, which yields

$$
\sum_{k=0}^{\infty} \frac{\xi^{-2 k}}{2 k-N+1}=-\xi^{1-N} \int_{0}^{\xi} \frac{1-w^{N}}{1-w^{2}} d w+\xi^{1-N} \int_{0}^{1 / \xi} \frac{1}{1-w^{2}} d w
$$

For the first series in (3.1) one obtains likewise

$$
\sum_{k=0}^{\infty} \frac{\xi^{-2 k}}{2 k+N+1}=\xi^{1+N} \int_{0}^{1 / \xi} \frac{w^{N}}{1-w^{2}} d w
$$


Adding these two expressions and multiplying by the factor $2 / \xi$ in (3.1) yields

$$
\begin{aligned}
S_{N}(z)=2 \xi^{-N} & \left(\int_{0}^{1 / \xi} \frac{1}{1-w^{2}} d w-\int_{0}^{\xi} \frac{1-w^{N}}{1-w^{2}} d w\right) \\
& +2 \xi^{N} \int_{0}^{1 / \xi} \frac{w^{N}}{1-w^{2}} d w .
\end{aligned}
$$

When $\xi \notin(-\infty,-1] \cup[1, \infty)$ the second integral on the right can be split into two to give

$$
S_{N}(z)=\xi^{-N}\left(\log \left(\frac{\xi+1}{\xi-1}\right)-\log \left(\frac{1+\xi}{1-\xi}\right)\right)+W_{N}(\xi)
$$

with $W_{N}(\xi)$ defined by (3.5). The term in parentheses is $+\pi i$ when $\operatorname{Im} \xi>0$ and $-\pi i$ when $\operatorname{Im} \xi<0$. This yields (3.3) in the case $N$ even. The formula for $N$ odd follows from (3.2).

When $\xi \in(-\infty,-1] \cup[1, \infty)$, the term in parentheses in (3.6) can be expressed as a principal value integral, namely

$$
\int_{0}^{1 / \xi} \frac{1}{1-w^{2}} d w-\int_{0}^{\xi} \frac{1-w^{N}}{1-w^{2}} d w=f_{0}^{\xi} \frac{w^{N}}{1-w^{2}} d w .
$$

This follows from standard formulas from the theory of singular integrals; see for example $[6$, Sect. 2.12.8].

Remark 1 The integrals in (3.5) can be evaluated for $N \geq 2$ with the recurrence

$$
\begin{aligned}
\int_{0}^{\xi} \frac{w^{N}}{1-w^{2}} d w & =\int_{0}^{\xi} \frac{w^{N-2}\left(w^{2}-1+1\right)}{1-w^{2}} d w \\
& =-\frac{1}{N-1} \xi^{N-1}+\int_{0}^{\xi} \frac{w^{N-2}}{1-w^{2}} d w
\end{aligned}
$$

which yields, for even $N$,

$$
\int_{0}^{\xi} \frac{w^{N}}{1-w^{2}} d w=\frac{1}{2} \log \left(\frac{1+\xi}{1-\xi}\right)-\sum_{k=1}^{N / 2} \frac{\xi^{2 k-1}}{2 k-1} .
$$

By inserting this formula (and the analogous one with $\xi$ replaced by $\xi^{-1}$ ) into (3.5) one can express the function $W_{N}(\xi)$ also in terms of finite sums rather than integrals. By using the identity $T_{N}(z)=\left(\xi^{N}+\xi^{-N}\right) / 2$ the corresponding formula for $S_{N}(z)$ can be reduced to the finite sum formula of Lemma 4.

Remark 2 Alternatively, the integrals in (3.5) can also be expressed in terms of special functions. The software package Maple yields

$$
\int_{0}^{\xi} \frac{w^{N}}{1-w^{2}} d w=\frac{1}{2} \xi^{N+1} \Phi\left(\xi^{2}, 1, \frac{1}{2}(N+1)\right),
$$

where $\Phi$ is the Lerch transcendent; see [7, Sect. 1.11]. Therefore,

$$
W_{N}(\xi)=\xi \Phi\left(\xi^{2}, 1, \frac{1}{2}(N+1)\right)+\xi^{-1} \Phi\left(\xi^{-2}, 1, \frac{1}{2}(N+1)\right) .
$$


Mathematica, on the other hand, produces

$$
\int_{0}^{\xi} \frac{w^{N}}{1-w^{2}} d w=\frac{1}{N+1} \xi^{N+1} F_{1}\left(1, \frac{1}{2}(N+1) ; \frac{1}{2}(N+3) ; \xi^{2}\right),
$$

where ${ }_{2} F_{1}$ is the Gauss hypergeometric function; see [7, Ch. II]. (The equivalence of the $\Phi$ and ${ }_{2} F_{1}$ representations follows from formula (10) in [7, Sect. 1.11].) For numerical computations these formulas should be used with care, however, as the special functions are multi-valued.

Remark 3 Concerning large $N$ behaviour, asymptotic expansions of the integrals in (3.5) can be derived by repeated integration by parts. Alternatively, one could use the connection with the $\Phi$ function summarized in (3.7). López and Ferreira derived in [8, Thm. 1] a full asymptotic expansion, including error term, for the $\Phi$ function in the situation where its third argument is large. This theorem can be applied directly to obtain the full asymptotic behaviour of $W_{N}(\xi)$, but we just cite leading order behaviour here. For the case $N$ even and $\xi \notin(-\infty,-1] \cup[1, \infty)$,

$$
W_{N}(\xi)=-4 \frac{\left(\xi^{2}+1\right) \xi}{\left(\xi^{2}-1\right)^{2}} N^{-2}+O\left(N^{-4}\right),
$$

relevant for Fejér, and

$$
W_{N}(\xi)-W_{N-2}(\xi)=16 \frac{\left(\xi^{2}+1\right) \xi}{\left(\xi^{2}-1\right)^{2}} N^{-3}+O\left(N^{-5}\right),
$$

relevant for Clenshaw-Curtis. For odd $N$ the formulas on the right should be multiplied by $1 / z$. Note that these formulas cease to be valid when $\xi$ is near \pm 1 .

Having obtained the asymptotics of $S_{N}(z)$ we can now return to the error formulas given in Theorem 2. The asymptotic behaviour of the other quantities in that theorem is well-known and can be summarized as follows: For $z \in E_{\varrho}$ with $N \rightarrow \infty$,

$$
\begin{aligned}
T_{N}(z) & \sim 2^{-1} \xi^{N} \\
P_{N}(z) & \sim \pi^{-1 / 2}(2 N)^{-1 / 2}\left(z^{2}-1\right)^{-1 / 4} \xi^{N+1 / 2} \\
Q_{N}(z) & \sim \pi^{1 / 2}(2 N)^{-1 / 2}\left(z^{2}-1\right)^{-1 / 4} \xi^{-(N+1 / 2)}
\end{aligned}
$$

see $[13$, Ch. VIII]. (For the conditions of validity of the latter two approximations we refer to [13, p. 188].)

Comparing the three error formulas in Theorem 2 one concludes that the denominators all have essentially the same behaviour, namely $O\left(\varrho^{N}\right)$, with $\varrho=|\xi|$. Any differences in convergence rate, as seen in Figure 1 for example, will therefore have to come from the numerators. That is, we need to compare the behaviour of $Q_{N}(z), S_{N}(z)$, and $S_{N}(z)-S_{N-2}(z)$, as $N \rightarrow \infty$. By the above asymptotic estimates these quantities behave like $O\left(\varrho^{-N}\right), O\left(N^{-2}\right)$ and $O\left(N^{-3}\right)$. Together with the factor $O\left(\varrho^{-N}\right)$ contributed by the 
denominator, this indicates convergence rates of $O\left(\varrho^{-2 N}\right), O\left(N^{-2} \varrho^{-N}\right)$, and $O\left(N^{-3} \varrho^{-N}\right)$, respectively, for Gauss, Fejér, and Clenshaw-Curtis quadrature. (More precise formulas are given in Theorem 7 below.) These convergence rates are consistent with Figure 1 (where $\varrho \approx 1.28$ ). We remark that these results are not new-similar convergence estimates can be found for example in [3, Sect. IV.6].

What is new, however, is the factor $\pm \xi^{-N} \pi i$ in Lemma 6. This transient term is responsible for producing the kinks in Figure 1. The asymptotic estimates $O\left(N^{-2} \varrho^{-N}\right)$ and $O\left(N^{-3} \varrho^{-N}\right)$ mentioned in the previous paragraph are obtained under the assumption that the term $\pm \xi^{-N} \pi i$ in (3.3) is negligible compared to the factors involving the $W_{N}(\xi)$ function (which are of leading order $O\left(N^{-2}\right)$ or $O\left(N^{-3}\right)$ ). When $|\xi|$ is near 1 , however, this term may not be negligible and it may in fact dominate. Therefore, for $N$ less than some critical value the convergence rates do not look like $O\left(N^{-2} \varrho^{-N}\right)$ or $O\left(N^{-3} \varrho^{-N}\right)$ but rather like $O\left(\varrho^{-2 N}\right)$ in both the Fejér and Clenshaw-Curtis cases, which is essentially the convergence rate of Gauss quadrature.

The kink in Figure 1 is therefore characterized by the fact that the $\pm \xi^{-N} \pi i$ term in Lemma 6 is of the same magnitude as the terms involving the $W_{N}(\xi)$ function. For a given $\xi$ the kink is accordingly located at the value of $N$ that satisfies

$$
\text { Fejér: } \quad \pi|\xi|^{-N}=\left|W_{N}(\xi)\right|,
$$

and

$$
\text { Clenshaw-Curtis: } \pi\left|1-\xi^{2}\right||\xi|^{-N}=\left|W_{N}(\xi)-W_{N-2}(\xi)\right| .
$$

With $\xi=\frac{1}{4}(1+\sqrt{17}) i \approx 1.28 i$ a numerical solution of these equations yields approximately $N=36.83$ (Fejér) and $N=54.17$ (Clenshaw-Curtis). These values pinpoint the locations of the kinks quite well as we have seen in Figure 1. For odd $N$ these two formulas should be modified according to the factor in the right-hand side of (3.3). In this case we get $N=29.40$ (Fejér) and $N=46.74$ (Clenshaw-Curtis). These values are consistent with the observation that for odd $N$ the kink is located at smaller values of $N$ than for even $N$.

For computations such as these one has to be able to evaluate $W_{N}$. Several computational strategies suggest themselves: numerical quadrature applied to the integrals in (3.5), the finite sum formulas mentioned in Remark 1, and the special functions of Remark 2. For the computations mentioned in the previous paragraph we have used (3.7) and Maple's function LerchPhi. For the computations of the next section, however, we have computed the $\Phi$ function in MATLAB using its series expansion (formula (1) in [7, Sect. 1.11]), the convergence of which we accelerated with the epsilon algorithm. As an alternative to these numerical approaches one could, for large $N$, replace the right-hand sides of (3.10)-(3.11) with their leading order asymptotic approximations as given in Remark 3. This strategy yields approximations to the critical values of $N$ that differ only in the second decimal digit from those given in Figure 1.

The large $N$ approximation also enables one to extract asymptotic information on the location of the kink. For example, if one assumes $z=\epsilon i$, with $0<\epsilon \ll 1$, one can use asymptotic iteration rather than numerical methods to solve (3.10)-(3.11) (with right-hand sides replaced by the leading terms in (3.8)-(3.9)). Thus one finds that the 
critical values of $N$ are related to $\epsilon$ by

$$
\epsilon \sim \frac{3 \log N}{N}, \text { and } \epsilon \sim \frac{4 \log N}{N}, \quad N \rightarrow \infty,
$$

respectively, for Fejér and Clenshaw-Curtis. These details, and other properties of the kink phenomenon, are presented in the next section.

We conclude by summarizing the asymptotic behaviour of the error formulas of Theorem 2 .

Theorem 7 With the notation of Theorem 2 and $\xi \notin(-\infty,-1] \cup[1, \infty)$, the $N \rightarrow \infty$ behaviour of the quadrature errors is given by

$$
I\left(f_{z}\right)-G_{N}\left(f_{z}\right) \sim 2 \pi \xi^{-2 N-1},
$$

and, for $N$ even,

$$
I\left(f_{z}\right)-F_{N}\left(f_{z}\right) \sim-8 \frac{\left(\xi^{2}+1\right)}{\left(\xi^{2}-1\right)^{2}} \frac{\xi^{1-N}}{N^{2}}, \quad I\left(f_{z}\right)-C_{N}\left(f_{z}\right) \sim 32 \frac{\left(\xi^{2}+1\right)}{\left(\xi^{2}-1\right)^{3}} \frac{\xi^{3-N}}{N^{3}} .
$$

(For odd $N$ an additional factor $1 / z$ should multiply the right-hand sides.) For $N$ less than the critical value defined by (3.10)-(3.11), however, the latter two errors behave instead like

$$
I\left(f_{z}\right)-F_{N}\left(f_{z}\right) \approx 2 i \sigma \pi \xi^{-2 N}, \quad I\left(f_{z}\right)-C_{N}\left(f_{z}\right) \approx-2 i \sigma \pi \xi^{2-2 N}
$$

which is essentially the same rate as for Gauss quadrature. (Here $\sigma= \pm 1$ as defined by (3.4).)

\section{The Kink Curve}

In the previous section we fixed $z$ (and $\xi$ ) and computed the corresponding value of $N$ that defines the kink. One can look at this from the other direction: fix $N$ and ask what $z$ this corresponds to. This determines a curve in the complex $z$-plane, defined by (3.10)-(3.11), that we shall call the kink curve. That is, for any value of $z$ inside the curve the Fejér and Clenshaw-Curtis rules are about as accurate as the Gauss rule for that particular value of $N$.

In Figure 2 we plot these curves for $N=16$. Also shown, as the dots, are the roots of the function $\phi(z)-r_{N}(z)$ as defined in (2.8). (In the Clenshaw-Curtis case these roots have also been plotted in [15].) As in [15], a winding number argument can be used to establish that there are 16 (resp. 14) finite roots for the Fejér (resp. ClenshawCurtis) approximation when $N=16$. We remind the reader that because of $(2.2)$, (2.5), and (2.8), the roots of $\phi(z)-r_{N}(z)$ are located exactly on this curve; there is no approximation here (other than machine roundoff error).

In order to obtain more information on these curves, we now switch to the asymptotic approximations summarized in Remark 3. We show details only for the Fejér case. (The analysis for Clenshaw-Curtis is analogous and we omit the particulars.) 
(a) Fejer

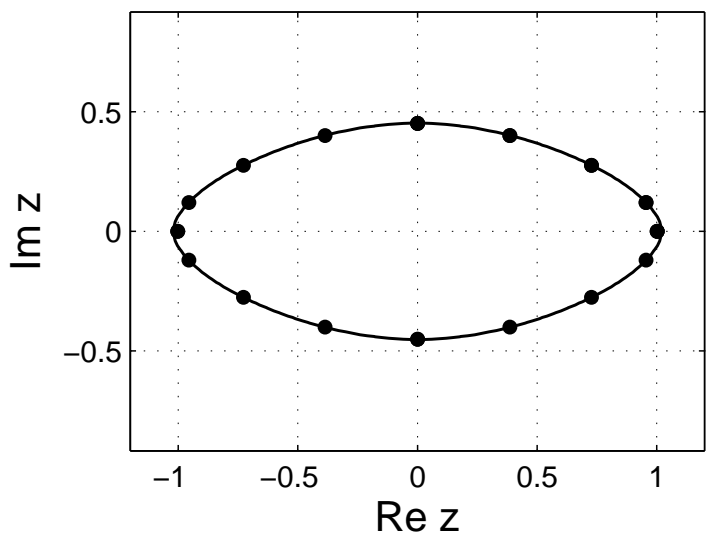

(b) Clenshaw-Curtis

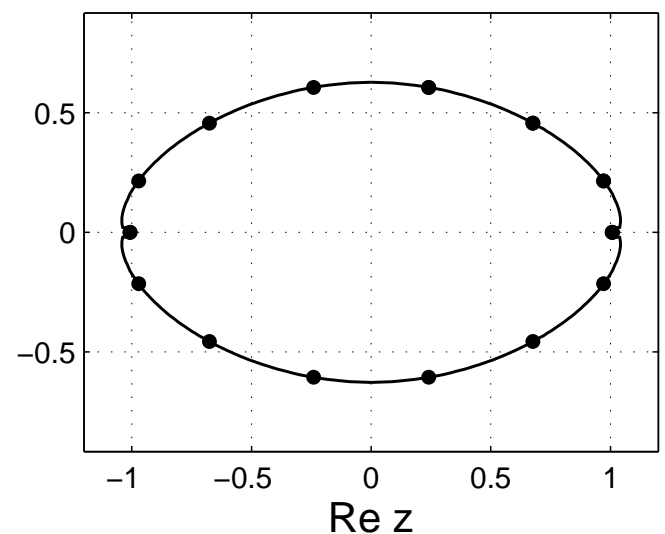

Figure 2: The kink curves as defined by (3.10)-(3.11), with $N=16$. The dots are the roots of $\phi(z)-r_{N}(z)$ defined by (2.8), or equivalently, the roots of $E_{N}(z)$ defined by $(2.2)$.

For reasons that will become clear shortly, it is best to start with the case $N$ odd. In this case the definition of the kink curve (3.10) is modified to

$$
\pi|\xi|^{-N}=|2 z|^{-1}\left|W_{N+1}(\xi)+W_{N-1}(\xi)\right|
$$

where we have used (3.3). Using the leading term in the asymptotic approximation (3.8) we find that this curve is given to first order by

$$
\pi|\xi|^{-N}=8 N^{-2}\left|\xi-\xi^{-1}\right|^{-2}
$$

We investigate this with an informal perturbation analysis. Near the interval $z \in$ $[-1,1]$, assume

$$
\xi=e^{i \theta}(1+\epsilon), \quad 0 \leq \theta \leq 2 \pi,
$$

where $|\epsilon| \ll 1$. We compute

$$
\xi-\xi^{-1}=2(i \sin \theta+\epsilon \cos \theta)+O\left(\epsilon^{2}\right)
$$

and therefore (4.2) can be approximated by

$$
\pi|1+\epsilon|^{-N}=2 N^{-2}|i \sin \theta+\epsilon \cos \theta|^{-2} .
$$

By taking logarithms and disregarding constants like $\log \pi$ and $\log 2$ in comparison to $\log N$, one gets

$$
N \log |1+\epsilon|=2 \log N+2 \log |i \sin \theta+\epsilon \cos \theta| .
$$

The term involving $\theta$ can be neglected in comparison to the other two terms, except when $\theta=O\left(N^{-1}\right)$ (and similarly for $\theta$ near $\pi$ and $2 \pi$ ). But $\theta \rightarrow 0, \pi, 2 \pi$ implies that $\xi$ is near \pm 1 , and the asymptotic approximations that we have used so far cease to be 
valid (see the last sentence of Remark 3). We shall deal with this situation separately. Therefore, ignoring the $\theta$ term on the right and using the approximation $\log |1+\epsilon| \sim \epsilon$ on the left yields the estimate

$$
\epsilon \sim \frac{2 \log N}{N}
$$

Away from $\xi= \pm 1$ we have therefore obtained the approximation

$$
\xi=e^{i \theta}\left(1+\frac{2 \log N}{N}\right)
$$

Switching to the variable $z=x+i y$, it means that the kink curve can be approximated by

$$
z=\cos \theta+i\left(\frac{2 \log N}{N}\right) \sin \theta
$$

which defines the ellipse

$$
x^{2}+\left(\frac{N}{2 \log N}\right)^{2} y^{2}=1 .
$$

In the Clenshaw-Curtis case this is modified to

$$
x^{2}+\left(\frac{N}{3 \log N}\right)^{2} y^{2}=1 .
$$

Figure 3 shows that these two approximations match the actual kink curves (3.10)-(3.11) very well.

(a) Fejer

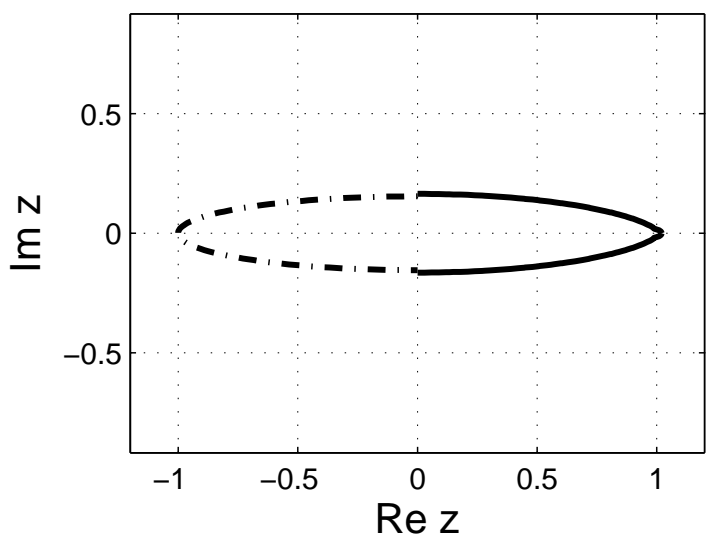

(b) Clenshaw-Curtis

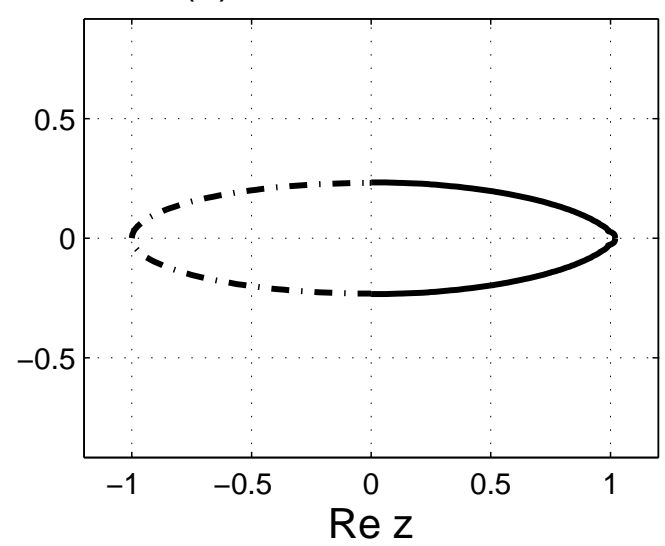

Figure 3: The kink curves (3.10)-(3.11) are shown as the solid curves in the right half of each figure; the approximate ellipses (4.5)-(4.6) derived here are the dash-dot curves on the left. Note the good agreement. Here $N=51$.

Equations (4.5)-(4.6) are not to be compared with the estimates (3.12), as those were obtained under the assumption that $N$ is even. In this case the perturbation analysis is slightly different. Again we present the details only for the Fejér case. 
For $N$ even the formula (4.2) becomes

$$
\pi|\xi|^{-N}=4 N^{-2}\left|\xi+\xi^{-1}\right|\left|\xi-\xi^{-1}\right|^{-2} .
$$

Proceeding as in (4.3)-(4.4) we see that (4.4) has to be modified to

$$
N \log |1+\epsilon|=2 \log N+2 \log |i \sin \theta+\epsilon \cos \theta|-\log |\cos \theta+\epsilon i \sin \theta| .
$$

The final term on the right is new. This term cannot be neglected in comparison with the terms containing $N$ when $\theta=\pi / 2+O\left(N^{-1}\right.$ ) (and similarly for $\theta$ near $3 \pi / 2$ ). This means that the ellipse (4.5) ceases to be a valid approximation to the kink curve when $z$ is near the imaginary axis.

Near $\theta=\pi / 2$ and $3 \pi / 2$ eq. (4.7) can be approximated by

$$
N \epsilon=2 \log N-\log |\epsilon| \text {. }
$$

Substitute into this equation the ansatz

$$
\epsilon \sim \frac{c \log N}{N}
$$

with $c$ a positive constant. This yields, to leading order, $c=3$, and for Clenshaw-Curtis, $c=4$. This is how the estimates (3.12) were derived.

Figure 4 shows the curves (3.10)-(3.11) for a large, even value of $N$. As predicted, the kink curves deviate from the approximating ellipses (4.5)-(4.6) near the imaginary axes, resulting in little "humps" of width $O\left(N^{-1}\right)$ on the curves. In the Fejér case (resp. Clenshaw-Curtis), the approximating ellipse (4.5) (resp. (4.6)) intersects the positive imaginary axis at $z=2 i \log N / N(\operatorname{resp} .3 i \log N / N)$. The kink curve, however, turns away towards $z=+i \infty$ and intersects the imaginary axis at the larger value $z=3 i \log N / N$ (resp. $4 i \log N / N)$, approximately, as predicted by our analysis. Figure 4 also explains why the kinks in Figure 1 occur at larger values of $N$ when $N$ is even.

The final topic we would like to address is the situation near $z= \pm 1$. Numerical experiments reveal that the curve intersects the real axis at

$$
x=1+O\left(N^{-2}\right)
$$

(and its negative). We now offer heuristic arguments leading to the value of the implied constant on the right.

Let the positive constants $\alpha$ and $\beta$ be defined by

$$
x \sim 1+\alpha N^{-2}, \quad \xi \sim 1+\beta N^{-1},
$$

where, to leading order, $\alpha=\beta^{2} / 2$. This ansatz for $\xi$ is inserted into the formula for $S_{N}(x)$ given in Lemma 6 . The value of $\beta$ follows from setting the leading terms in the asymptotic expansion of $S_{N}(x)$ to zero.

We begin with the second integral in (3.5),

$$
2 \xi^{N} \int_{0}^{1 / \xi} \frac{w^{N}}{1-w^{2}} d w=2 \xi \int_{0}^{1} \frac{u^{N}}{\xi^{2}-u^{2}} d u
$$


(a) Fejer

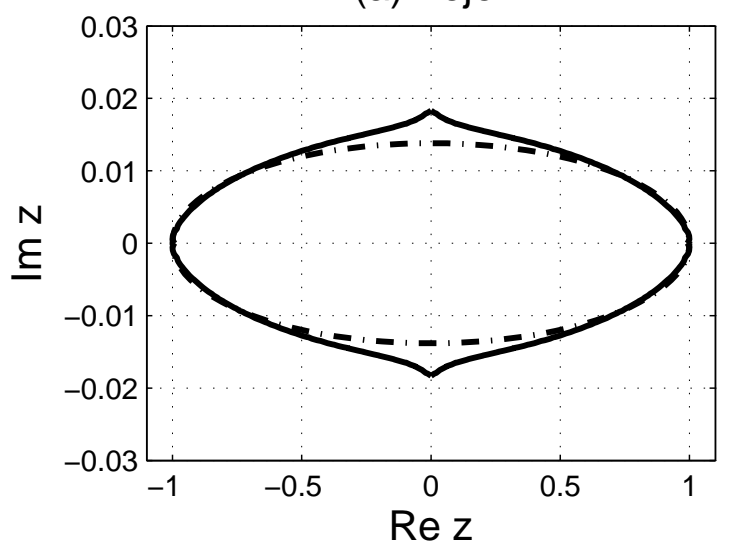

(b) Clenshaw-Curtis

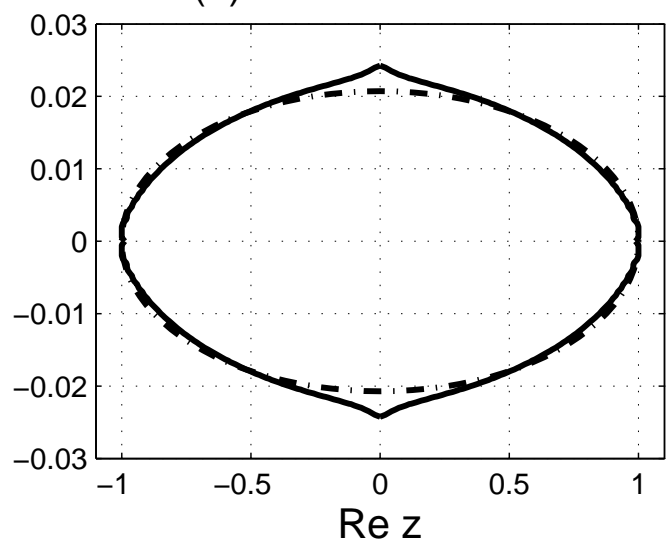

Figure 4: The kink curves (3.10)-(3.11) are shown as solid curves, the approximating ellipses (4.5)-(4.6) as dash-dot curves. Near the imaginary axis there is a deviation between the two as explained below (4.7). This occurs for even values of $N$; here $N=1000$. (Note that in this figure we have used unequal scales on the real and imaginary axes to reveal more detail; in Figures $2-3$ the scales were equal.)

where we have substituted $u=\xi w$. The integrand on the right can be expanded in partial fractions involving $1 /(\xi-u)$ and $1 /(\xi+u)$. Since $\xi \rightarrow 1$ as $N \rightarrow \infty$, the leading contribution will come from the term involving $1 /(\xi-u)$, and therefore

$$
2 \xi^{N} \int_{0}^{1 / \xi} \frac{w^{N}}{1-w^{2}} d w \sim \int_{0}^{1} \frac{u^{N}}{\xi-u} d u .
$$

Let $\xi \sim 1+\beta N^{-1}$ and define $s$ by $u=1-s N^{-1}$. Then, using the fact that $(1-s / N)^{N} \rightarrow$ $e^{-s}$ as $N \rightarrow \infty$, one obtains

$$
2 \xi^{N} \int_{0}^{1 / \xi} \frac{w^{N}}{1-w^{2}} d w \sim \int_{0}^{\infty} \frac{e^{-s}}{\beta+s} d s=e^{\beta} E_{1}(\beta),
$$

where $E_{1}$ is the exponential integral. A similar calculation produces for the first integral in $(3.5)$

$$
2 \xi^{-N} f_{0}^{\xi} \frac{w^{N}}{1-w^{2}} d w \sim-e^{-\beta} \operatorname{Ei}(\beta) .
$$

(Our notation for $E_{1}$ and $\mathrm{Ei}$ is consistent with the definitions in [2, Ch. 5].)

Substitute (4.9) and (4.10) into $S_{N}(x)=0$. This gives, to leading order, the following equation for $\beta$ :

$$
e^{\beta} E_{1}(\beta)-e^{-\beta} \operatorname{Ei}(\beta)=0 .
$$

A numerical solution produces, to eight place accuracy,

$$
\beta=0.87908775, \quad \alpha=\frac{1}{2} \beta^{2}=0.38639764 .
$$


We offer the following table as numerical verification of this result. The values of $x$ shown in the middle column are the numerically computed roots of $S_{N}(x)$, or equivalently, the roots of $\phi(x)-r_{N}(x)$ in the Fejér case.

\begin{tabular}{ccc}
$N$ & $x$ & $(x-1) N^{2}$ \\
\hline 32 & 1.00037708 & 0.386129 \\
64 & 1.00009432 & 0.386330 \\
128 & 1.00002358 & 0.386381 \\
256 & 1.00000590 & 0.386393
\end{tabular}

A similar analysis for Clenshaw-Curtis quadrature would require higher order asymptotics, something we have not pursued. Numerical results suggest that the value of $\alpha$ is about 1.73 in this case.

Acknowledgements. We are indebted to David Elliott for correcting several mistakes in earlier drafts of this paper. Elliott also showed us an independent, contour integration derivation of an estimate for $S_{N}(z)$ that is similar to our Lemma 6 combined with the asymptotics of Remark 3. Chelo Ferreira and José Lopez, authors of [8], generously shared their knowledge of the Lerch $\Phi$ function. Milton Maritz helped with Mathematica and Walter Gautschi brought reference [12] to our attention.

\section{References}

[1] M. J. Ablowitz and A. S. Fokas, 2003. Complex variables: introduction and applications. Cambridge University Press, Cambridge, second edition.

[2] M. Abramowitz and I. A. Stegun, 1970. Handbook of mathematical functions with formulas, graphs, and mathematical tables. Dover Publications, Inc., New York.

[3] H. Brass, 1977. Quadraturverfahren. Vandenhoeck \& Ruprecht, Göttingen. Studia Mathematica, Skript 3.

[4] M. M. Chawla, 1968. Error estimates for the Clenshaw-Curtis quadrature. Math. Comp., 22: 651-656.

[5] P. J. Davis, 1975. Interpolation and approximation. Dover Publications Inc., New York.

[6] P. J. Davis and P. Rabinowitz, 1984. Methods of numerical integration. Academic Press Inc., Orlando, FL, second edition.

[7] A. Erdélyi, W. Magnus, F. Oberhettinger and F. G. Tricomi, 1953. Higher transcendental functions. Vol. I. McGraw-Hill Book Company, Inc., New York.

[8] C. Ferreira and J. L. López, 2004. Asymptotic expansions of the Hurwitz-Lerch zeta function. J. Math. Anal. Appl., 298. 
[9] W. Gautschi, 1981. A survey of Gauss-Christoffel quadrature formulae. In E. B. Christoffel (Aachen/Monschau, 1979), pages 72-147. Birkhäuser, Basel.

[10] V. I. Krylov, 1962. Approximate calculation of integrals. The Macmillan Co., New York.

[11] J. C. Mason and D. C. Handscomb, 2003. Chebyshev polynomials. Chapman \& Hall/CRC, Boca Raton, FL.

[12] S. E. Notaris, 2006. Integral formulas for Chebyshev polynomials and the error term of interpolatory quadrature formulae for analytic functions. Math. Comp., 75: $1217-1231$.

[13] G. Szegö, 1975. Orthogonal polynomials. American Mathematical Society, Providence, R.I., fourth edition. American Mathematical Society, Colloquium Publications, Vol. XXIII.

[14] H. Takahasi and M. Mori, 1971. Estimation of errors in the numerical quadrature of analytic functions. Applicable Anal., 1: 201-229.

[15] L. N. Trefethen, 2006. Is Gauss quadrature better than Clenshaw-Curtis? SIAM Review. Submitted.

[16] J. A. C. Weideman and D. P. Laurie, 2000. Quadrature rules based on partial fraction expansions. Numer. Algorithms, 24: 159-178. 